\title{
A New Flux Observer and Simplified ADRC Controllers for SSTEKF Sensorless Vector Control of an Induction Motor
}

\author{
Haijun CHE, Ying TIAN, Jingming YANG*, Zitong ZHAO \\ Engineering Research Center, Ministry of Education for Intelligent Control Systems and Intelligent Equipment, \\ Yanshan University, Hebei province, Qinhuangdao, 066000, China \\ hjche@ysu.edu.cn, ysuty2018@163.com,yangjmysu@outlook.com (*Corresponding author), \\ zitongzhao24@gmail.com
}

\begin{abstract}
A novel flux observer is proposed with a view to improving the stability and reliability of the sensorless vector control system of an induction motor, which is robust with regard to different parameters and features no phase lag or attenuation. At the same time PI controllers are replaced in the double-closed loop system with an active disturbance rejection controller (ADRC), which reduces overshoot when speed changes and further improves the steady-state performance of the system. Finally, the method based on the Symmetric Strong Tracking Extended Kalman Filter (SSTEKF) is employed for speed parameter identification with the purpose of improving the traditional EKF double- closed loop speed sensorless system in order to further enhance the robustness and anti-interference performance of the system. The simulation results confirm the good performance of the proposed technique.
\end{abstract}

Keywords: Induction motor, Sensorless speed control, Vector control, Flux linkage observer, Symmetric strong tracking extended Kalman filter, Active disturbance rejection controller.

\section{Introduction}

Sensorless control of induction motors is an attractive technology that has gained considerable market share in the past few years (Chen et al., 2019). This phenomenon has prompted many scholars to study speed sensorless control technology, and put forward many practical and effective methods, such as sliding mode observer (SMO) (Saadaoui et al., 2015) adaptive fullorder observer (AFO) (Sun et al., 2016), model reference adaptive system (MRAS) (Cao et al., 2018), high frequency signal injection method (Liu et al., 2005), extended kalman filter (EKF) (Hongxia et al., 2013), etc.

The difference between sliding mode (SM) variable structure control and conventional control lies in the fact that the former is a variable structure system (Kim, Lee \& Lee, 2016). According to the current state of the system, by changing the structure of the system, the system is forced to follow a predetermined trajectory in response, which is meant to make the system stable and robust. But its steady-state performance is poor, and some noise always exists because of the switch function. Sun et al. (2016) proposed some of the new rules for the self-adaption of proportional integral controller gains so as to obtain a higher performance (Bui et al., 2018). Chen et al. (2014) and Maksoud et al. (2019) focused on the robustness of AFO against motor parameter variations, they focused on solving the problem that of speed fluctuating more when the speed is smaller. The MRAS structure is simple and easy to understand, but its accuracy is low. Due to its pure integral link, error accumulation and DC- drift will occur, and the reference model cannot accurately reflect the state of the click itself (Zhang, Cheng \& Zhang, 2018). The signal injection method is meant to calculate the detected stator current to obtain the information related to the magnetic salient pole, and the stator current value must be accurate, which results in the poor anti-noise ability of the algorithm, and the identification accuracy is closely related to the frequency range of the injected signal (Guglielmi et al., 2017). Therefore, the accuracy of speed identification is poor in practical use, and it is not applicable at low speed. In comparison with other methods, if the covariance between the measured noise and the noise inside the system is known, the EKF can optimize the noise (Hrbáč et al., 2016). Moreover, if the rotor speed is added to the dynamic model of induction motor, the nonlinear state model can be linearized by extended Kalman filter in order to make the state model available in the estimation process. In recent years, the induction motor drive system based on extended Kalman filter (EKF) has made great progress. Since EKF can estimate the state of the system accurately when the system contains noise, it is very suitable for motor state observation (Taheri, 2012). However, EKF has poor anti-mutation ability and anti-interference ability. In general, the EKF is biased by state estimation, and its robustness of model mismatch is very poor, and the state estimation problem of the nonlinear system is often met in practical system design problems. Therefore, to improve the EKF and make 
it have a better performance has become an urgent research topic. Many literatures have investigated this problem. In Zaky (2012), EKF-based speed sensorless estimation algorithm is put forward for the stator-oriented and rotor-oriented models of asynchronous motor. Taheri \& Zhalebaghi (2017) added predictive control on this basis. However, there are still problems such as poor robustness and poor anti-interference ability. In (Yin et al., 2016), a speed sensorless STEKF control based on the least square algorithm is proposed (Liu et al., 2017). A fading factor was added to the original algorithm, that is to say, more information is used for improving the tracking performance. Moreover, the noise suppression is better than the EKF (Zhang, Ma $\&$ Gao, 2018). In addition, in direct vector control system of IM, rotor flux observation is a key link to realize accurate magnetic field orientation, which directly affects the precision of the control system (In'kov et al., 2018). The traditional expression of the equation of stator current in fixed coordinate system method and the expression of the equation of stator voltage in fixed coordinate system method have some disadvantages (Holtz, 2002). The former is greatly affected by the volatility of IM parameters. When temperature rise or frequency change of the motor will affect the rotor resistance, the magnetic saturation degree will affect mutual inductance and rotor inductance, which will lead to the distortion of flux amplitude and position (Holtz, 2006). Although the latter is less affected by the change of motor parameters, because the expression of the equation of stator voltage in fixed coordinate system contains pure integral terms, the initial value of integral and the accumulated error all affect the calculation result (Xiao et al., 2021). In case of the lower speed operation, the change of stator resistance pressure drop also has a great influence (Xu et al., 2014).

In practical applications, PI control method is usually employed for adjusting the current loops, speed loop and flux linkage loop (Schauder, 1989). At the same time, although the vector control can accomplish the decoupling control of flux and torque, the method based on the mathematical model is still affected by operation conditions and parameter mismatch. Among the existing modern control methods, sliding mode control method, internal mode control method, adaptive control method, predictive control method and so on have made some progress in the research of induction motor variable frequency control. However, even under normal working conditions, the sliding mode control used the sign function, so it will cause large electromagnetic torque ripples when the induction motor is running at low speed. The intrinsic time of the internal model controller is invariable, which features a contradiction between robustness and rapidity. The adaptive control is complicated and this approach requires a high level of processing power. ADRC is a kind of controller with good effect in nonlinear systems. By real-time estimation and compensation of internal and external disturbances and in combination with nonlinear control strategy, the system can obtain better robustness and dynamic performance.

Here, a new flux observer is first presented as an ideal flux observation model for estimating the flux of IM sensorless control, which has no lag or no attenuation and also no position error, and the PI controller is improved to the ADRC controller, which effectively reduces the system overshoot. In this paper, an improved speed observer based on SSTEKF speed estimation method is presented. The correctness and feasibility of this method are proved by the improved flux linkage stability and simulation of the whole motor control system. This paper is arranged as follows. Section 2 presents the SSTEKF algorithm which is applied to the induction motor speed sensorless control system. Section 3 sets forth a new flux observer used in the induction motor speed sensorless control system. Section 4 presents the simulation results for the proposed method. Section 5 concludes this paper.

\section{Process of EKF and SSTEKF Observer for IM}

\subsection{Process of EKF}

The dynamic electrical model of a three-phase IM speed sensorless control has four state variables, namely stator current and rotor flux (Aydogmus and Talu, 2012). In the speed sensorless control, the speed is unknown. In order to identify the speed, it is also regarded as a state variable. The corresponding augmented motor model is obtained as follows:

$$
\begin{aligned}
& \hat{\boldsymbol{x}}_{k}=\boldsymbol{A}_{k} \boldsymbol{x}_{k-1}+\boldsymbol{B}_{k} \boldsymbol{u}_{k-1}+\boldsymbol{G}_{k} \boldsymbol{v}_{k-1}, \\
& \hat{\boldsymbol{y}}_{k}=\boldsymbol{C}_{k} \hat{\boldsymbol{x}}_{k}+\boldsymbol{w}_{k} \\
& \boldsymbol{u}_{k}=\left(\begin{array}{lllll}
\boldsymbol{u}_{s \alpha, k} & \boldsymbol{u}_{s \beta, k}
\end{array}\right)^{T} \\
& \boldsymbol{x}_{k}=\left(\begin{array}{lllll}
i_{s \alpha, k} & i_{s \beta, k} & \psi_{r \alpha, k} & \psi_{r \beta, k} & \omega_{r, k}
\end{array}\right)^{T}
\end{aligned}
$$




$$
\begin{aligned}
& \boldsymbol{A}_{k}=\left[\begin{array}{ccccc}
1-\frac{K_{r}}{K_{l}} M & 0 & \frac{L_{m} R_{r}}{L_{r}^{2} K_{l}} M & \frac{P L_{m} \omega_{0}^{(n)}}{2 L_{r} K_{l}} M & 0 \\
0 & 1-\frac{K_{r}}{K_{l}} M & \frac{P L_{m} \omega_{0}^{(n)}}{2 L_{r} K_{l}} M & \frac{L_{m} R_{r}}{L_{r}^{2} K_{l}} M & 0 \\
\frac{L_{m}}{T_{r}} M & 0 & 1-\frac{1}{T_{r}} M & -\frac{P}{2} \omega_{0}^{(n)} M & 0 \\
0 & \frac{L_{m}}{T_{r}} M & \frac{P}{2} \omega_{0}^{(n)} M & 1-\frac{1}{T_{r}} M & 0 \\
0 & 0 & 0 & 0 & 1
\end{array}\right] \\
& \boldsymbol{B}_{k}=\left[\begin{array}{cc}
\frac{M}{K_{l}} & 0 \\
0 & \frac{M}{K_{l}} \\
0 & 0 \\
0 & 0 \\
0 & 0
\end{array}\right] \\
& \boldsymbol{C}_{k}=\left[\begin{array}{lllll}
1 & 0 & 0 & 0 & 0 \\
0 & 1 & 0 & 0 & 0
\end{array}\right] \\
& K_{l}=\sigma L_{s}, K_{r}=R_{s}+L_{m}^{2} R_{r} / L_{r}^{2}, \sigma=1-L_{m}{ }^{2} /\left(L_{r} L_{s}\right) \text {, }
\end{aligned}
$$

where $M$ is sampling time. $\mathrm{P}$ is the number of magnetic poles. $\omega_{0}^{(n)}$ is rotor speed. $G_{k}$ is weighted noise matrix $Q$ and $R$ are the covariance matrixes of the noises, respectively. They are represented as: $Q=\operatorname{cov}(v)=E\{v, v\}, R=\operatorname{cov}(w)=E\{w, w\}$.

The specific process includes the following two processes:

1) Prediction process:

$$
\begin{aligned}
& \tilde{\boldsymbol{x}}_{k}=\boldsymbol{A}_{k-1} \boldsymbol{x}_{k-1}+\boldsymbol{B}_{k-1} \boldsymbol{u}_{k-1}, \\
& \tilde{\boldsymbol{P}}_{k}=\boldsymbol{G}_{k} \hat{\boldsymbol{P}}_{k-1} \boldsymbol{G}_{k}^{T}+\boldsymbol{Q}_{k-1} .
\end{aligned}
$$

where " $\sim$ "is the predicted value, $G_{k}$ is calculated as follows

$$
\begin{aligned}
\boldsymbol{G}_{k} & =\left.\left(\frac{\partial}{\partial x}\left(\boldsymbol{A}_{k} \boldsymbol{x}_{k}+\boldsymbol{B}_{k} \boldsymbol{u}_{k-1}\right)\right)\right|_{x=\tilde{x}_{k}} \\
& =\left[\begin{array}{ccccc}
1-\frac{K_{r}}{K_{l}} M & 0 & \frac{L_{m} R_{r}}{L_{r}^{2} K_{l}} M & \frac{P L_{m} \omega_{0}^{(n)}}{2 L_{r} K_{l}} M & \frac{M L_{m}}{K_{l} L_{r}} \hat{\psi}_{r \beta, k} \\
0 & 1-\frac{K_{r}}{K_{l}} M & \frac{P L_{m} \omega_{0}^{(n)}}{2 L_{r} K_{l}} M & \frac{L_{m} R_{r}}{L_{r}^{2} K_{l}} M & -\frac{M L_{m}}{K_{l} L_{r}} \hat{\psi}_{r \alpha, k} \\
\frac{L_{m}}{T_{r}} M & 0 & 1-\frac{1}{T_{r}} M & -\frac{P}{2} \omega_{0}^{(n)} M & -M \hat{\psi}_{r \beta, k} \\
0 & \frac{L_{m}}{T_{r}} M & \frac{P}{2} \omega_{0}^{(n)} M & 1-\frac{1}{T_{r}} M & M \hat{\psi}_{r \alpha, k} \\
0 & 0 & 0 & 0 & 1
\end{array}\right]
\end{aligned}
$$

where " $\hat{\psi}_{r \alpha, k} "$ is the estimated value of the rotor flux.

Kalman gain:

$$
\boldsymbol{K}_{k}=\tilde{\boldsymbol{P}}_{k} \boldsymbol{H}_{k}^{T}\left(\boldsymbol{H}_{k} \tilde{\boldsymbol{P}}_{k} \boldsymbol{H}_{k}^{T}+\boldsymbol{R}_{k}\right)^{-1}
$$

2) Filtering process:

Update status prediction variable:
$\hat{\boldsymbol{x}_{k}}=\tilde{\boldsymbol{x}_{k}}+\boldsymbol{K}_{k}\left(\boldsymbol{y}_{k}-\boldsymbol{H}_{k} \tilde{\boldsymbol{x}_{k}}\right)$

Update the error covariance matrix:

$\hat{\boldsymbol{P}}_{k}=\left(\boldsymbol{I}-\boldsymbol{K}_{k} \boldsymbol{H}_{k}\right) \tilde{\boldsymbol{P}}_{k}$

where "^" is the updated value.

The induction motor sensorless vector control overall block diagram of the control system is shown in Figure 1.

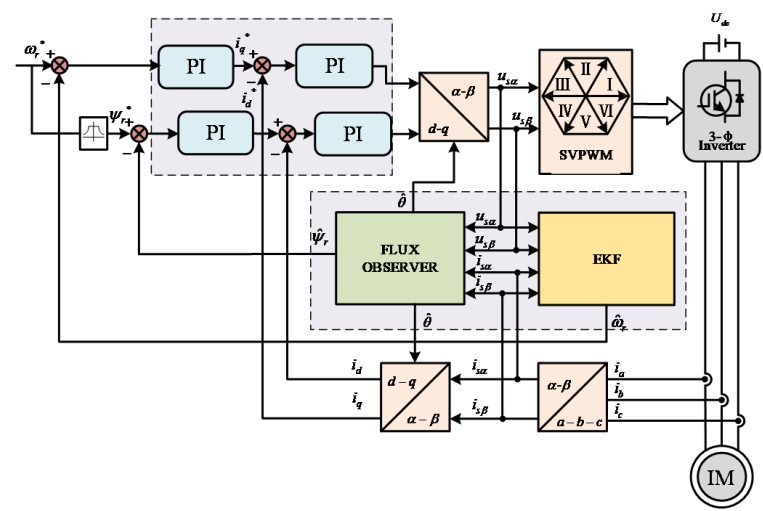

Figure 1. Diagram of the original speed sensorless system

Since $K_{k}$ and $P_{k}$ are fixed values, their tracking ability to deal with sudden changes is not strong, and they cannot deal with changes of external factors quickly. Moreover, model accuracy depends on the values of $Q$ and $R$, but they are also fixed in $\mathrm{EKF}$, which requires that one knows the external noise model accurately in order to determine it.

\subsection{Process of SSTEKF}

In SSTEKF, according to the orthogonality principle, when there is a large model mismatch, time-varied gain should be adjusted online so that the output residual sequences are orthogonal to each other, which also indicates that all effective information in the residual sequence has been extracted. It meets the following conditions:

$$
\begin{aligned}
& E\left(\left[x_{k}-\hat{x}_{k}\right]\left[x_{k}-x_{k}\right]^{T}\right)=\min \\
& E\left(\varepsilon_{k} \varepsilon_{k+j}^{T}\right)=0 j=1,2 \ldots
\end{aligned}
$$

where $\varepsilon_{k}$ is residual sequence. In equation (4), multiple suboptimal fading factors are introduced, and when different data channels are fading at different rates, filters with higher performance energy can be obtained. Then, the expression of the covariance matrix of the predicted state is:

$\tilde{\boldsymbol{P}_{k}}=\boldsymbol{\Lambda}_{k} \boldsymbol{G}_{k} \hat{\boldsymbol{P}_{k-1}} \boldsymbol{G}_{k}^{T}+\boldsymbol{Q}_{k-1}$

$\Lambda_{k}=\operatorname{diag}\left\{\lambda_{k}^{1}, \lambda_{k}^{2}, \ldots, \lambda_{k}^{n}\right\}$ 
$\lambda_{k}^{i}=\left\{\begin{array}{cc}\beta_{i} c_{k}, & \beta_{i} c_{k}>1 \\ 1, & \beta_{i} c_{k} \leq 1\end{array}\right.$

$c_{k}=\operatorname{tr}\left[\boldsymbol{N}_{k}\right] /\left(\sum_{i=1}^{n} \beta_{i} \boldsymbol{M}_{k, i i}\right)$

$\boldsymbol{N}_{k}=\boldsymbol{V}_{k}-\boldsymbol{R}_{k}-\boldsymbol{H}_{k} \boldsymbol{Q}_{k-1} \boldsymbol{H}_{k}^{T}$

$\boldsymbol{M}_{k}=\boldsymbol{G}_{k} \hat{\boldsymbol{P}_{k-1}} \boldsymbol{G}_{k}^{T} \boldsymbol{H}_{k}^{T} \boldsymbol{H}_{k}$

$\boldsymbol{V}_{k}=\left\{\begin{array}{cc}\varepsilon_{1} \varepsilon_{1}^{T}, & k=1 \\ \frac{\rho \boldsymbol{V}_{k-1}+\varepsilon_{k} \varepsilon_{k}^{T}}{1+\rho}, & k \geq 2\end{array}\right.$

where $\Lambda_{k}$ is fading matrix, $\rho(0<\rho \leq 1)$ is the forgetting factor. $\lambda_{k}^{i} \equiv 1$ when it is known from prior knowledge that some component $\left(x_{k}^{i}\right)$ of $x_{k}$ will not mutate, which will speed up the calculation of fading factors. When no prior knowledge is available, $\beta_{i}=1(i=1, \cdots, n)$. To avoid the asymmetry and the control system divergence due to $\Lambda_{k}$, the error covariance matrix is developed as:

$\tilde{P}_{k}=\Lambda_{k} G_{k} \hat{P_{k-1}} G_{k}^{T} \bar{\Lambda}_{k}+Q_{k-1}$

According to Cholesky's triangular theory, the expression of $\bar{\Lambda}_{k}$ can be written as:

$\Lambda_{k}=\bar{\Lambda}_{k} \bar{\Lambda}_{k}^{T}$

$\bar{\Lambda}_{k}=\operatorname{diag}\left\{\sqrt{\lambda_{k}^{1}}, \sqrt{\lambda_{k}^{2}}, \ldots, \sqrt{\lambda_{k}^{n}}\right\}$

The SSTEKF is better than EKF because the residual sequences are orthogonal to each other, and the gain matrix is tuned online by introducing the fading factors into the covariance matrix of the predicted state. With the EKF reaching steady state, the error covariance matrix and gain are already quite small and cannot be tracked in time for subsequent mutations. Therefore, an attenuation factor is introduced in order to reduce the influence of old data on the estimated state and estimated performance.

\section{The Proposed Flux Linkage Observer}

\subsection{Theoretical Formulation}

Stable and accurate flux linkage observations are especially important in induction motor speed sensorless speed control (Myoung-Ho et al., 1998). However, the methods of calculating flux by stator voltage model and the stator current model commonly used in traditional induction motor speed regulation process are inaccurate and unstable (Aydogmus \& Suenter, 2012).
In order to improve the accuracy and robustness of flux linkage estimation, the improved model uses a disturbance observer, which combines the expression of the equation of stator voltage in fixed coordinate system method and the expression of the equation of stator current in fixed coordinate system method. First, the stator voltage model is employed to estimate the flux linkage, then the flux is introduced into the current model which obtains the current estimate. The error between the measured current and the estimated current are used as a disturbance feedback to the expression of the equation of stator voltage in fixed coordinate system method to improve the accuracy and robustness of the estimated flux linkage. The equation of the current model is:

$\psi_{r \alpha}=\frac{1}{T_{r} s+1}\left(L_{m} i_{s \alpha}-\omega T_{r} \psi_{r \beta}\right)$

$\psi_{r \beta}=\frac{1}{T_{r} s+1}\left(L_{m} i_{s \beta}+\omega T_{r} \psi_{r \alpha}\right)$

where $\omega$ is the rotor speed of the motor. The expression of the equation of stator voltage in fixed coordinate system for calculating rotor flux in $\alpha-\beta$ coordinate system is written as

$\psi_{r \alpha}=\frac{L_{r}}{L_{m}}\left[\int\left(u_{s \alpha}-R_{s} i_{s \alpha}\right) d t-\sigma L_{s} i_{s \alpha}\right]$

$\psi_{r \beta}=\frac{L_{r}}{L_{m}}\left[\int\left(u_{s \beta}-R_{s} i_{s \beta}\right) d t-\sigma L_{s} i_{s \beta}\right]$

The expression of Laplace transform is

$\psi_{r \alpha}=\frac{L_{r}}{L_{m}}\left[\frac{1}{S}\left(u_{s \alpha}-R_{s} i_{s \alpha}\right)-\sigma L_{s} i_{s \alpha}\right]$

$\psi_{r \beta}=\frac{L_{r}}{L_{m}}\left[\frac{1}{s}\left(u_{s \beta}-R_{s} i_{s \beta}\right)-\sigma L_{s} i_{s \beta}\right]$.

Figure 2 illustrates a block diagram of the proposed flux observer, where $\hat{\psi}_{r \alpha}$ is the rotor flux linkage of induction motor in $\alpha$ axis. The subscripts $\alpha$ and $\beta$ of the parameters in the figure represent each parameter in the stationary $\alpha-\beta$ reference frame. The currents estimated by the expression of the equation of stator voltage in fixed coordinate system are:

$\left\{\begin{array}{l}\hat{i}_{s \alpha}=\frac{\hat{\psi}_{r \alpha}\left(T_{r} s+1\right)+\omega T_{r} \psi_{r \beta}}{L_{m}} \\ \hat{i}_{s \beta}=\frac{\hat{\psi}_{r \beta}\left(T_{r} s+1\right)-\omega T_{r} \psi_{r \alpha}}{L_{m}}\end{array}\right.$

The key to solving the problem is to consider the errors between $i_{\alpha \beta}$ and $i_{\alpha \beta}$ as disturbances. 


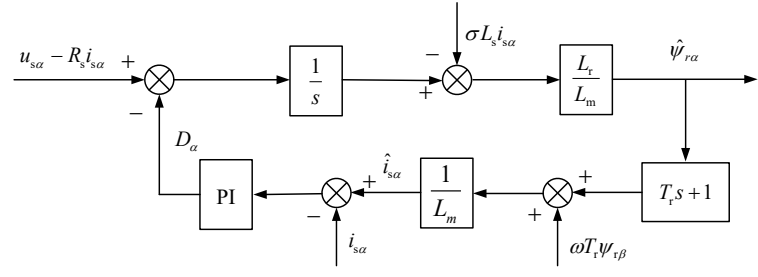

Figure 2. Diagram of the flux observer

In Figure 2, the adjustable parameter $G$ is introduced as linear compensation in the system. $D_{\alpha}$ is the feedback brought into the expression equation of stator voltage in fixed coordinate system. And $\omega$ is the revolving speed of induction motor. Therefore, the transfer function of flux linkage is:

$$
\left\{\begin{aligned}
\hat{\psi}_{r \alpha} & =\frac{L_{r}}{L_{m}}\left[\frac{1}{s}\left(u_{s \alpha}-R_{s} i_{s \alpha}-D_{\alpha}\right)-\sigma L_{s} i_{s \alpha}\right] \\
& =\frac{L_{r}}{L_{m}}\left\{\frac{1}{s}\left[u_{s \alpha}-R_{s} i_{s \alpha}-G\left(\hat{i}_{s \alpha}-i_{s \alpha}\right)\right]-\sigma L_{s} i_{s \alpha}\right\} \\
\hat{\psi}_{r \beta} & =\frac{L_{r}}{L_{m}}\left[\frac{1}{s}\left(u_{s \beta}-R_{s} i_{s \beta}-D_{\beta}\right)-\sigma L_{s} i_{s \beta}\right] \\
& =\frac{L_{r}}{L_{m}}\left\{\frac{1}{s}\left[u_{s \beta}-R_{s} i_{s \beta}-G\left(\hat{i}_{s \beta}-i_{s \beta}\right)\right]-\sigma L_{s} i_{s \beta}\right\}
\end{aligned}\right.
$$

By substituting (25) into (26), the following can be obtained

$\left\{\begin{array}{l}\hat{\psi}_{r \alpha}=\frac{L_{r}}{L_{m}}\left\{-\frac{1}{s}\left[u_{s \alpha}-R_{s} i_{s \alpha}-G\left(\frac{\hat{\psi}_{r \alpha}\left(T_{r} s+1\right)+\omega T_{r} \psi_{r \beta}}{L_{m}}-i_{s \alpha}\right)\right]-\sigma L_{s} i_{s \alpha}\right\} \\ \hat{\psi}_{r \beta}=\frac{L_{r}}{L_{m}}\left\{-\frac{1}{s}\left[u_{s \beta}-R_{s} i_{s \beta}-G\left(\frac{\hat{\psi}_{r \beta}\left(T_{r} s+1\right)-\omega T_{r} \psi_{r \alpha}}{L_{m}}-i_{s \beta}\right)\right]-\sigma L_{s} i_{s \beta}\right\}\end{array}\right.$

By rearranging (27), the transfer function between the estimated flux and the respective variable signals could be written as:

$\left\{\begin{array}{l}\hat{\psi}_{r \alpha}=\frac{L_{m}\left(u_{s \alpha}-R_{s} i_{s \alpha}-\sigma L_{s} i_{s \alpha} s+G i_{s \alpha}\right)-G \omega T_{r} \psi_{r \beta}}{\left(\frac{L_{m}{ }^{2}}{L_{r}}+G T_{r}\right) s+G} \\ \hat{\psi}_{r \beta}=\frac{L_{m}\left(u_{s \beta}-R_{s} i_{s \beta}-\sigma L_{s} i_{s \beta} s+G i_{s \beta}\right)+G \omega T_{r} \psi_{r \alpha \alpha}}{\left(\frac{L_{m}{ }^{2}}{L_{r}}+G T_{r}\right) s+G}\end{array}\right.$

The transfer function of $G$ is:

$$
G(s)=k_{p}+\frac{k_{i}}{s}
$$

Let us set $k_{p}=\omega_{c} L_{m}$ and $k_{i}=\omega_{c}^{2} L_{m}$, then the Laplace expressions of the estimated rotor flux equations of the IM in the stationary $\alpha-\beta$ coordinate system are given as:

$$
\begin{gathered}
\hat{\psi}_{r \alpha}=\frac{\frac{L_{r}}{L_{m}}\left(\frac{u_{s \alpha}-R_{s} i_{s \alpha}}{s}-\sigma L_{s} i_{s \alpha}\right)+\frac{L_{r}}{L_{m}}\left(\frac{\omega_{c}}{s}+\frac{\omega_{c}^{2}}{s}\right)\left(T_{r} s+1\right)+\frac{L_{m} i_{s \alpha}-\omega T_{r} \psi_{r \beta}}{T_{r} s+1}}{1+\frac{L_{r}}{L_{m}}\left(\frac{\omega_{c}}{s}+\frac{\omega_{c}^{2}}{s}\right)\left(T_{r} s+1\right)} \\
\hat{\psi}_{r \beta}=\frac{\frac{L_{r}}{L_{m}}\left(\frac{u_{s \beta}-R_{s} i_{s \beta}}{s}-\sigma L_{s} i_{s \beta}\right)+\frac{L_{r}}{L_{m}}\left(\frac{\omega_{c}}{s}+\frac{\omega_{c}^{2}}{s}\right)\left(T_{r} s+1\right)+\frac{L_{m} i_{s \beta}+\omega T_{r} \psi_{r \alpha}}{T_{r} s+1}}{1+\frac{L_{r}}{L_{m}}\left(\frac{\omega_{c}}{s}+\frac{\omega_{c}^{2}}{s}\right)\left(T_{r} s+1\right)}
\end{gathered}
$$

The cut-off frequency of the new flux linkage observer is denoted by $\omega_{c}$. Using (19), (20), (23) and (24), the estimated flux function (30) becomes (31) as it is shown below

$$
\left\{\begin{array}{l}
\hat{\psi}_{r \alpha}=\psi_{r \alpha} \\
\hat{\psi}_{r \beta}=\psi_{r \beta}
\end{array}\right.
$$

It's not hard to derive the conclusion that the estimated flux linkages error is zero in equation (31). If the jamming signals (such as $u_{s \alpha}, u_{s \beta}, i_{s \alpha}$, $i_{s \beta}, L_{r}, L_{m}, R_{r}, R_{s}$ and $\theta$ ) exist, the improved flux observer will be more steady. By the Laplace transform, it is obvious that the transfer functions are structurally similar to low-pass filters and bandpass filters. That is to say, the new flux linkage model has the resistance to high-frequency noises and low-frequency noises. Hence, the improved flux linkage model is adopted to improve the performance of speed sensorless vector control.

\subsection{Stability Analysis}

To carry out the stability analysis at steady state for flux linkages, the flux linkages transfer functions in stationary reference frame can be expressed as:

$\left\{\begin{array}{l}\hat{\psi}_{r \alpha}=\psi_{r \alpha}+E r r_{\alpha} \\ \hat{\psi}_{r \beta}=\psi_{r \beta}+E r r_{\beta}\end{array}\right.$

where $E r r_{\alpha}$ and $\operatorname{Err}_{\beta}$ are the errors between the actual flux and estimated flux. It is necessary to prove that if $\mathrm{Err}_{\alpha}$ and $\mathrm{Err}_{\beta}$ are steady, then the proposed observer is steady.

The stability analysis of $E r r_{\alpha}$ and $E r r_{\beta}$ for sensorless control of IM drive by using RouthHurwitz Criterion ensures the stability of the scheme as the $D(s)$ are found to be positive in this case.

When $u_{s \alpha}$ or $u_{s \beta}$ encounters interferences

$$
\left\{\begin{array}{l}
\operatorname{Err}_{\alpha}=\frac{s}{\left(\omega_{c} T_{r}+\frac{L_{m}}{L_{r}}\right) s^{2}+\left(\omega_{c}+\omega_{c}^{2} T_{r}\right) s+\omega_{c}^{2}} \Delta u_{s \alpha} \\
\operatorname{Err}_{\beta}=\frac{s}{\left(\omega_{c} T_{r}+\frac{L_{m}}{L_{r}}\right) s^{2}+\left(\omega_{c}+\omega_{c}^{2} T_{r}\right) s+\omega_{c}^{2}} \Delta u_{s \beta}
\end{array}\right.
$$

The closed loop transfer function of equation (33) is:

$$
G(s)=\frac{s}{\left(\omega_{c} T_{r}+\frac{L_{m}}{L_{r}}\right) s^{2}+\left(\omega_{c}+\omega_{c}^{2} T_{r}\right) s+\omega_{c}^{2}}
$$


The characteristic equation is:

$D(s)=\left(\omega_{c} T_{r}+\frac{L_{m}}{L_{r}}\right) s^{2}+\left(\omega_{c}+\omega_{c}^{2} T_{r}\right) s+\omega_{c}^{2}$

The Routh array table is given as:

\begin{tabular}{|c|c|c|}
\hline$s^{2}$ & $\omega_{c} T_{r}+\frac{L_{m}}{L_{r}}$ & $\omega_{c}^{2}$ \\
\hline$s^{1}$ & $\omega_{c}+\omega_{c}^{2} T_{r}$ & 0 \\
\hline$s^{0}$ & $\omega_{c}^{2}$ & 0 \\
\hline
\end{tabular}

where the parameters $T_{r}, L_{m}$ and $L_{r}$ are considered as positive constants in a sampling period. So, the coefficients of the polynomial $D(s)$ are all positive. It is known from Routh criterion that if the coefficients in the first column of Routh array table are all positive, the system is stable, and the global asymptotic stability of the observer with voltage interferences is guaranteed. Secondly, when $i_{\alpha \beta}$ encounters changes, the transfer functions of $E r r_{\alpha}$ and $E r r_{\beta}$ are:

$$
\left\{\begin{array}{l}
\operatorname{Err}_{\alpha}=\frac{-\sigma L_{s} s^{2}+\omega_{c} L_{m} s+\omega_{c}^{2} L_{m}}{\left(\omega_{c} T_{r}+\frac{L_{m}}{L_{r}}\right) s^{2}+\left(\omega_{c}+\omega_{c}^{2} T_{r}\right) s+\omega_{c}^{2}} \Delta i_{s \alpha} \\
\operatorname{Err}_{\beta}=\frac{-\sigma L_{s} s^{2}+\omega_{c} L_{m} s+\omega_{c}^{2} L_{m}}{\left(\omega_{c} T_{r}+\frac{L_{m}}{L_{r}}\right) s^{2}+\left(\omega_{c}+\omega_{c}^{2} T_{r}\right) s+\omega_{c}^{2}} \Delta i_{s \beta}
\end{array}\right.
$$

The closed loop transfer function is:

$$
G(s)=\frac{-\sigma L_{s} s^{2}+\omega_{c} L_{m} s+\omega_{c}^{2} L_{m}}{\left(\omega_{c} T_{r}+\frac{L_{m}}{L_{r}}\right) s^{2}+\left(\omega_{c}+\omega_{c}^{2} T_{r}\right) s+\omega_{c}^{2}}
$$

where the parameters $T_{r}, L_{m}$ and $L_{r}$ are considered as positive constants in a sampling period. The characteristic equation of the control system is as follows:

$$
D(s)=\left(\omega_{c} T_{r}+\frac{L_{m}}{L_{r}}\right) s^{2}+\left(\omega_{c}+\omega_{c}^{2} T_{r}\right) s+\omega_{c}^{2}
$$

The equation (38) is identical to the equation (35). Also, they are both based on the Routh Criterion. So the global asymptotic stability of the observer with current interferences is guaranteed. When the stator inductance $L_{s}$ has disturbances, the $E r r_{\alpha}$ and $\operatorname{Err}_{\beta}$ can be expressed as follows:

$$
\left\{\begin{array}{l}
\operatorname{Err}_{\alpha}=\frac{-i_{s \alpha} s^{2}}{\left(\omega_{c} T_{r}+\frac{L_{m}}{L_{r}}\right) s^{2}+\left(\omega_{c}+\omega_{c}^{2} T_{r}\right) s+\omega_{c}^{2}} \Delta L_{s} \\
\operatorname{Err}_{\beta}=\frac{-i_{s \beta} s^{2}}{\left(\omega_{c} T_{r}+\frac{L_{m}}{L_{r}}\right) s^{2}+\left(\omega_{c}+\omega_{c}^{2} T_{r}\right) s+\omega_{c}^{2}} \Delta L_{s}
\end{array}\right.
$$

The closed loop transfer function is:

$$
G(s)=\left\{\begin{array}{l}
\frac{-i_{s s} s^{2}}{\left(\omega_{c} T_{r}+\frac{L_{m}}{L_{r}}\right) s^{2}+\left(\omega_{c}+\omega_{c}^{2} T_{r}\right) s+\omega_{c}^{2}} \\
\frac{-i_{s} s^{2}}{\left(\omega_{c} T_{r}+\frac{L_{m}}{L_{r}}\right) s^{2}+\left(\omega_{c}+\omega_{c}^{2} T_{r}\right) s+\omega_{c}^{2}}
\end{array}\right.
$$

When the rotor resistance $R_{s}$ changes, the transfer functions of $\mathrm{Err}_{\alpha}$ and $\mathrm{Err}_{\beta}$ are:

$$
\left\{\begin{array}{l}
\operatorname{Err}_{\alpha}=\frac{-i_{s \alpha} s}{\left(\omega_{c} T_{r}+\frac{L_{m}}{L_{r}}\right) s^{2}+\left(\omega_{c}+\omega_{c}^{2} T_{r}\right) s+\omega_{c}^{2}} \Delta R_{s} \\
\operatorname{Err}_{\beta}=\frac{-i_{s \beta} s}{\left(\omega_{c} T_{r}+\frac{L_{m}}{L_{r}}\right) s^{2}+\left(\omega_{c}+\omega_{c}^{2} T_{r}\right) s+\omega_{c}^{2}} \Delta R_{s}
\end{array}\right.
$$

The transfer function of equation (41) is

$$
G(s)=\left\{\begin{array}{l}
\frac{-i_{s \alpha} s}{\left(\omega_{c} T_{r}+\frac{L_{m}}{L_{r}}\right) s^{2}+\left(\omega_{c}+\omega_{c}^{2} T_{r}\right) s+\omega_{c}^{2}} \\
\frac{-i_{s \beta} s}{\left(\omega_{c} T_{r}+\frac{L_{m}}{L_{r}}\right) s^{2}+\left(\omega_{c}+\omega_{c}^{2} T_{r}\right) s+\omega_{c}^{2}}
\end{array}\right.
$$

When the estimated $\theta$ has deviation, the transfer functions of $\mathrm{Err}_{\alpha}$ and $\mathrm{Err}_{\beta}$ are:

$$
\left\{\begin{array}{c}
\operatorname{Err}_{\alpha}=\frac{-\omega T_{r} \frac{L_{m}}{L_{r}} \psi_{f} \cos \theta\left(\omega_{c} s+\omega_{c}^{2}\right)}{\left(\omega_{c} T_{r}+\frac{L_{m}}{L_{r}}\right) s^{2}+\left(\omega_{c}+\omega_{c}^{2} T_{r}\right) s+\omega_{c}^{2}} \Delta \theta \\
\operatorname{Err}_{\beta}=\frac{-\omega T_{r} \frac{L_{m}}{L_{r}} \psi_{f} \sin \theta\left(\omega_{c} s+\omega_{c}^{2}\right)}{\left(\omega_{c} T_{r}+\frac{L_{m}}{L_{r}}\right) s^{2}+\left(\omega_{c}+\omega_{c}^{2} T_{r}\right) s+\omega_{c}^{2}} \Delta \theta
\end{array}\right.
$$

Considering $\sin \theta \Delta \theta$ or $\cos \theta \Delta \theta$ as a variate, the transfer function of equation(43) is as follows:

$$
G(s)=\left\{\begin{array}{c}
-\omega T_{r} \frac{L_{m}}{L_{r}} \psi_{f} \cos \theta\left(\omega_{c} s+\omega_{c}^{2}\right) \\
\frac{\left(\omega_{c} T_{r}+\frac{L_{m}}{L_{r}}\right) s^{2}+\left(\omega_{c}+\omega_{c}^{2} T_{r}\right) s+\omega_{c}^{2}}{\left(\omega T_{r} \frac{L_{m}}{L_{r}} \psi_{f} \sin \theta\left(\omega_{c} s+\omega_{c}^{2}\right)\right.} \\
\left(\omega_{c} T_{r}+\frac{L_{m}}{L_{r}}\right) s^{2}+\left(\omega_{c}+\omega_{c}^{2} T_{r}\right) s+\omega_{c}^{2}
\end{array}\right.
$$

The parameters $i_{\alpha \beta}$ in (40) and (42) and the parameter $\psi_{f}$ in (44) are treated as constant over a sampling period, which will not influence the stability performance of the control model. Therefore, the characteristic equation of these systems is:

$$
D(s)=\left(\omega_{c} T_{r}+\frac{L_{m}}{L_{r}}\right) s^{2}+\left(\omega_{c}+\omega_{c}^{2} T_{r}\right) s+\omega_{c}^{2}
$$


As it is indicated above, the characteristic equation $D(s)$ (45) is identical to equation (35), so they are both based on the Routh Criterion. Hence, the global asymptotic stability of the improved observer is guaranteed.

\section{Simulation}

In this section, the performance of the proposed model is verified in MATLAB/Simulink and its anti-interference performance is correlated with that of the improved model under the test cases bellow. The induction motor sensorless vector control overall block diagram of the new system is illustrated in Figure 3. The improvements are outlined with a dotted line. When the IM is powered by the three-phase voltage, the stator flux amplitude of the motor is constant, the space vector rotates at a constant speed, and the motion trajectory at the top of the flux vector is circular.

Figure 4 shows the flux circle changes from $0 \mathrm{~s}$ to $0.4 \mathrm{~s}$ before and after the improvement of the induction motor vector control model. It is not difficult to see that the improved model can quickly reach the stationary flux circle. This indicates that the control system can operate very well, which verifies the feasibility of the modified speed sensorless control system.

Figure 5 illustrates the speed tracking before and after the implementation of the improved model if a $10 \mathrm{~N} . \mathrm{m}$ load is applied suddenly. It fluctuates by about $1 \mathrm{rad} / \mathrm{s}$ when the load is suddenly added before improvement, but only fluctuates by about $0.5 \mathrm{rad} / \mathrm{s}$ after improvement, which indicates that the improved model has a high anti-interference ability.

Similarly, as it is shown in Figure 6, when a stator current of $2 \mathrm{~A}$ is suddenly applied to external elements at a certain moment, the tracked speed before and after the improvement is significantly different. The improved model featured a higher tracking performance when it encountered current mutation. In particular, according to a partial enlargement, in case of a single-phase current mutation, the peak value for the model before the improvement has reached $87 \mathrm{rad} / \mathrm{s}$, but only $84 \mathrm{rad} / \mathrm{s}$ after the improvement, which will reduce the loss for the motor in emergencies and improve the anti-interference ability of the system.

In addition, when the motor parameters change ( $L_{r}, R_{r}$ and $R_{s}$ ), as it is shown in Figures 7-9, the speed estimation of the improved model also features a higher tracking performance, that is, the improved model successfully solves the problem of low robustness in the sensorless control of IM.

To sum up, the simulation results ensure effectiveness of the improved model.

Table 1 lists the percentage of overshoot amount and settling time before and after the model improvement in the case of various parameter mutations. It can be seen from the table that the improved system features a strong antiinterference performance.

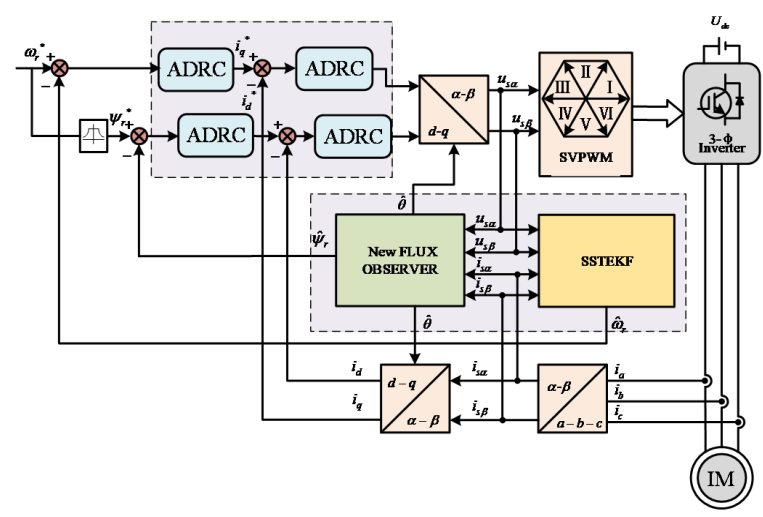

Figure 3. The structure of the improved induction motor sensorless vector control model
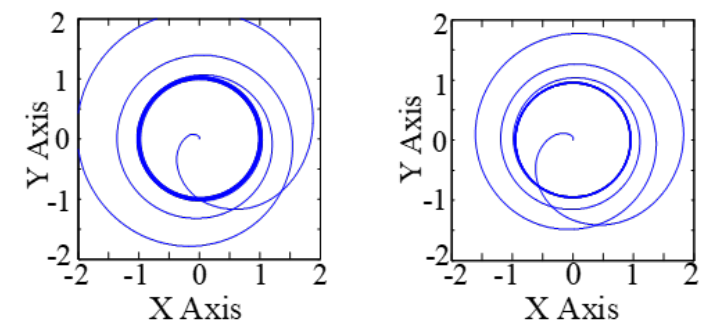

(a) before the implementation (b) after the implementation of the improved model of the improved model

Figure. 4. The flux circle before and after the implementation of the improbed induction motor sensorless vector control model

Table 1. Comparison of results before and after improvement

\begin{tabular}{cccccc|cccccc}
\hline model & \multicolumn{6}{c|}{ Overshoot(\%) } & \multicolumn{5}{c}{ Settling time(s) } \\
\hline & $i_{s \alpha}$ & $L_{m}$ & $R_{r}$ & $R_{s}$ & load & $i_{s \alpha}$ & $L_{m}$ & $R_{r}$ & $R_{s}$ & load \\
before & 8.75 & 87.6 & 7.5 & 10.1 & 1.00 & 0.022 & $\infty$ & 0.03 & 0.017 & 0.03 \\
after & 3.75 & 76.5 & 1.3 & 0.25 & 0.69 & 0.014 & 0.02 & 0.012 & 0 & 0.05 \\
\hline
\end{tabular}




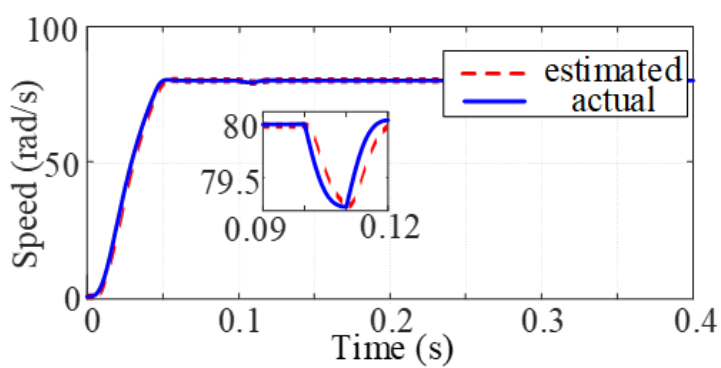

(a) before the implementation

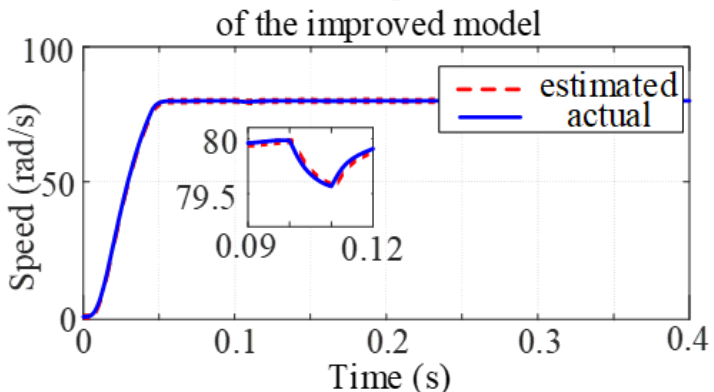

(b) after the implementation of the improved model

Figure 5. The estimated speed and actual speed when a 10 N.m load is applied suddenly

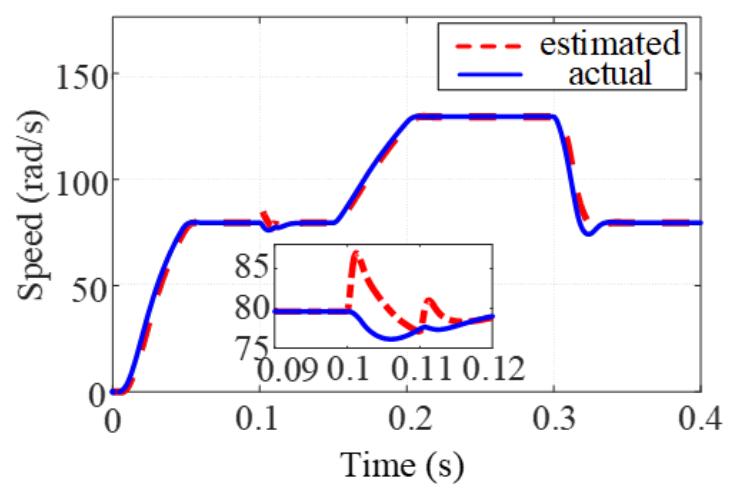

(a) before the implementation of the improved model

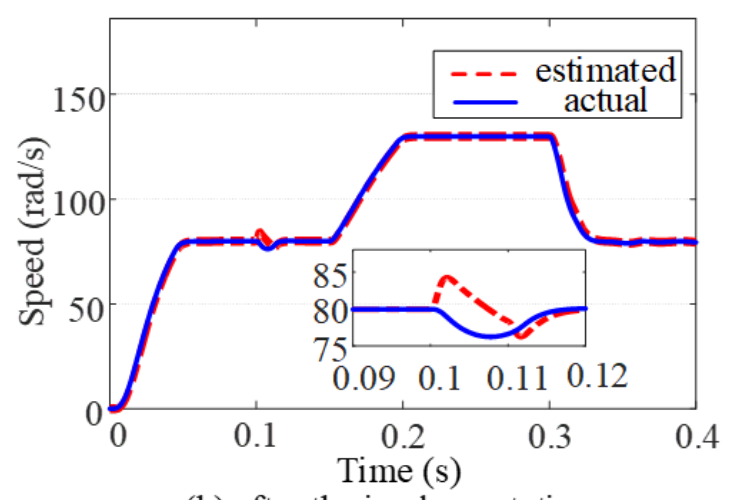

(b) after the implementation of the improved model

Figure 6. The estimated speed and actual speed when a 2 A current is applied suddenly

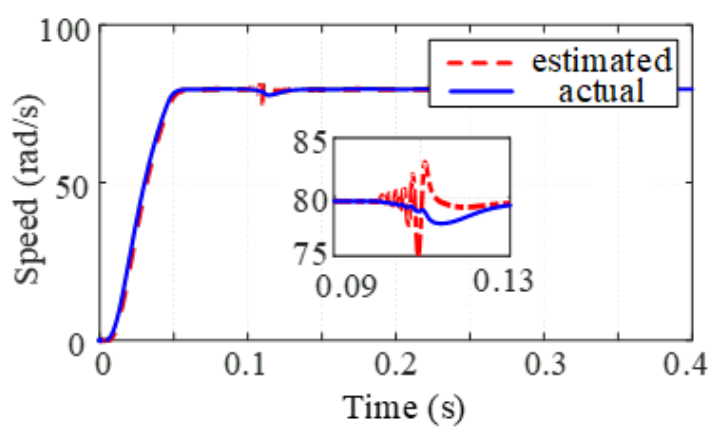

(a) before the implementation of the improved model

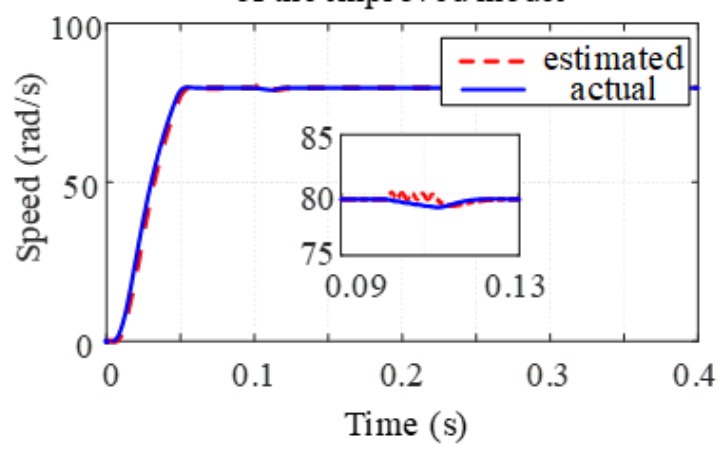

(b) after the implementation of the improved model

Figure 7. The estimated speed and actual speed when $R_{r}$ mutates to $2 R_{r}$

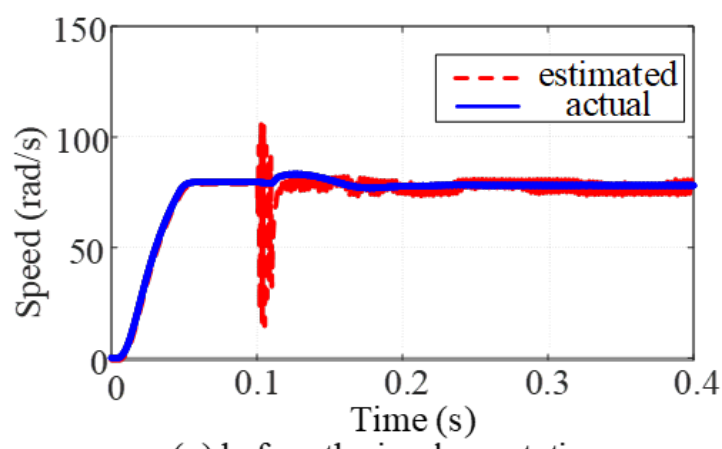

(a) before the implementation of the improved model

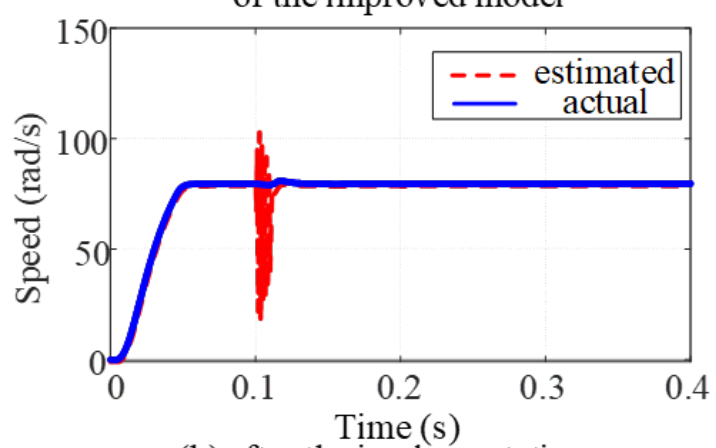

(b) after the implementation of the improved model

Figure 8. The estimated speed and actual speed when mutates to when $L_{r}$ mutates to $2 L_{r}$ 


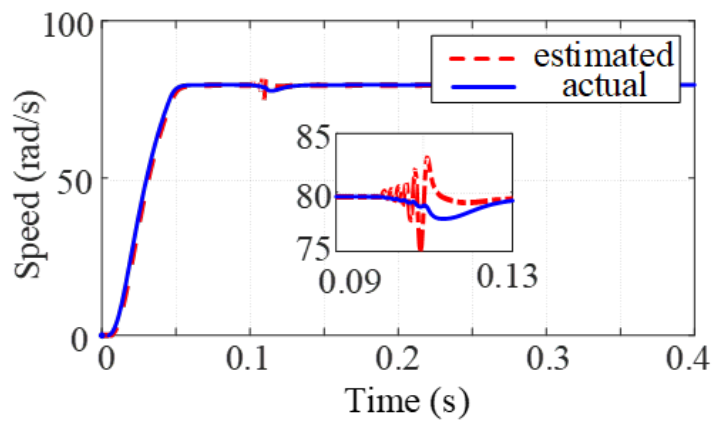

(a) before the implementation of the improved model

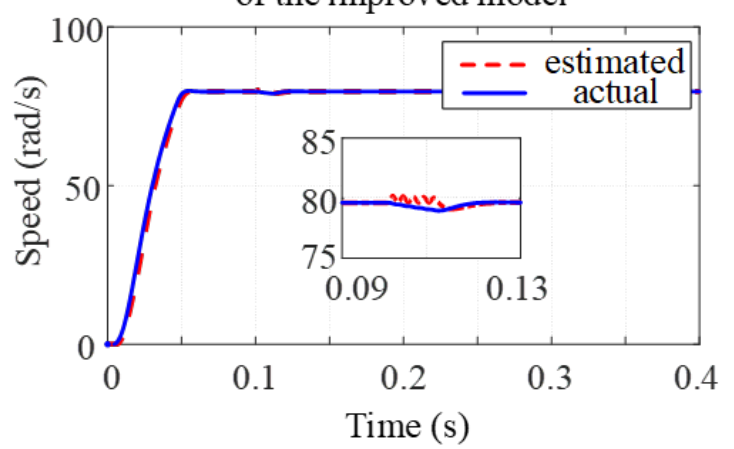

(b) after the implementation of the improved model

Figure 9. The estimated speed and actual speed when $R_{s}$ mutates to $2 R_{s}$

\section{REFERENCES}

Aydogmus, O. \& Suenter, S. (2012). Implementation of EKF based sensorless drive system using vector controlled PMSM fed by a matrix converter, International Journal of Electrical Power and Energy Systems, 43(1), 736-743.

Aydogmus, O. \& Talu, M. F. (2012). Comparison of Extended-Kalman- and Particle-Filter-Based Sensorless Speed Control, IEEE Transactions on Instrumentation and Measurement, 61(2), 402-410. DOI: 10.1109/TIM.2011.2164851

Bui, M. X., Rahman, M. F. \& Xiao, D. (2018). Sensorless Control of Interior Permanent Magnet Synchronous Machines Based on the Combination of the Modified FPE Method and SMO. In 2018 IEEE 9th International Symposium on Sensorless Control for Electrical Drives (SLED), 13-14 Sept (pp. 126-131).

Cao, P., Zhang, X., Yang, S., Xie, Z. \& Zhang, Y. (2018). Reactive-Power-Based MRAS for Online Rotor Time Constant Estimation in Induction Motor Drives, IEEE Transactions on Power Electronics, 33(12), 10835-10845.

Chen, B., Wang, T. Yao, W. \& Lee, K. (2014). Speed convergence rate-based feedback gains design of

\section{Conclusion}

This paper puts forward a new flux observer of IM based on SSTEKF and the ADRC controllers are employed for replacing all PI controllers of the speed sensorless control system. The correctness, stability and validity of the proposed method are verified by simulation on sensorless driver. Simulation results show that the new model is able to improve estimation accuracy, and reduce the modeling error. In comparison with the traditional voltage model method, this new flux linkage observer reduces the influence of estimated speed error and the variation of motor parameters, and improves the steadystate and transient performance of the system. Compared with the traditional model, this model has a better dynamic property and a lower overshoot at low speed under sudden loading. The results as depicted in Figures 5 to 8 confirm that the scheme preserves stability even under varying parameters. The problem of instability at impact load and parameter disturbance can be solved. However, as the accuracy of calculation is improved, the amount of calculation must be improved. The complexity of the system has increased to some extent. Due to the limitations of speed observation method, the effect of rotational speed may be not ideal at very low speed or zero speed.

adaptive full-order observer in sensorless induction motor drives, IET Electric Power Applications, 8(1), 13-22. DOI: 10.1049/iet-epa.2013.0210

Chen, J., Huang, J. \& Sun, Y. (2019). Resistances and Speed Estimation in Sensorless Induction Motor Drives Using a Model With Known Regressors, IEEE Transactions on Industrial Electronics, 66(4), 26592667. DOI:10.1109/TIE.2018.2849964

Guglielmi, P., Yousefi-talouki, A., Iabichino, G. \& Pellegrino, G. (2017). Sensorless direct torque control for PM-assisted synchronous motors with injection high-frequency signal into stator flux reference frame. In 2017 IEEE International Symposium on Sensorless Control for Electrical Drives (SLED), 18-19 Sept. (pp. 139-144).

Holtz, J. (2002). Sensorless control of induction motor drives, Proceedings of the IEEE, 90(8), 1359-1394. DOI: 10.1109/JPROC.2002.800726

Holtz, J. (2006). Sensorless Control of Induction Machines - With or Without Signal Injection?, IEEE Transactions on Industrial Electronics, 53(1), 7-30. DOI:10.1109/TIE.2005.862324 
Hongxia, Y., Xuan, W., Yanhong, W., Li, C. \& Quan, Z. (2013). Nonintrusive Efficiency Estimation of Induction Motor Using EKF. In Fifth International Conference on Measuring Technology and Mechatronics Automation (pp. 720-723). IEEE Computer Society. DOI: 10.1109/ICMTMA.2013.180

Hrbáč, Z., Sova, V. \& Grepl, R. (2016). Sensorless speed control of bldc motor using ekf with computed inputs and disturbance. Springer International Publishing. DOI: 10.1007/978-3-319-23923-1_7

In'kov, Y. M., Pudovikov, O. E. \& Pustovetov, M. Y. (2018). Characteristics of Output Filters Providing Electromagnetic Compatibility of the Frequency Converter of an Electric Drive with Asynchronous Motors, Russian Electrical Engineering, 89(9), 555-558.

Kim, J., Lee, Y. \& Lee, J. (2016). A sensorless speed estimation for indirect vector control of three-phase induction motor using Extended Kalman Filter. In 2016 IEEE Region 10 Conference (TENCON), 22-25 Nov. (pp. 3087-3090).

Liu, C. H., Chang, H. C. \& Wang, C. D. (2005). Very low speed sensorless control of induction motor drives using high-frequency signal injection, Journal of the Chinese Institute of Engineers, 28, 957-966.

Liu, M. Cui, N. Liu, S. Wang, C. Zhang, C. \& Gong, S. (2017). Adaptive strong tracking unscented Kalman filter based SOC estimation for lithium-ion battery. In 2017 Chinese Automation Congress, 20-22 Oct. (pp. 1437-1441).

Maksoud, H. A., Shaaban, S. M., Zaky, M. S. \& Azazi, H. Z. (2019). Performance and Stability Improvement of AFO for Sensorless IM Drives in Low Speeds Regenerating Mode, IEEE Transactions on Power Electronics, 34(8), 7812-7825.

Myoung-ho, S., Dong-seok, H., Soon-bong, C. \& Song-yul, C. (1998). An improved stator flux estimation for speed sensorless stator flux orientation control of induction motors. PESC 98 Record. In 29th Annual IEEE Power Electronics Specialists Conference (Cat. No.98CH36196), 22-22 May, vol. 15(2), (pp. 1581-1586).

Saadaoui, O., Khlaief, A., Abassi, M., Chaari, A. \& Boussak, M. (2015). Position sensorless vector control of PMSM drives based on SMO. In 2015 16th International Conference on Sciences and Techniques of Automatic Control and Computer Engineering (STA), 21-23 Dec. (pp. 545 - 550).

Schauder, C. (1989). Adaptive speed identification for vector control of induction motors without rotational transducers. In Conference Record of the IEEE Industry Applications Society Annual Meeting, 1-5 Oct., vol. 1 (pp. 493-499).
Sun, W., Yu, Y., Wang, G., Li, B. \& Xu, D. (2016). Design Method of Adaptive Full Order Observer With or Without Estimated Flux Error in Speed Estimation Algorithm, IEEE Transactions on Power Electronics, 31(3), 2609-2626.

Taheri, A. (2012). EKF modeling of field oriented control of six-phase induction motor, IEICE Electronics Express, 9(7), 642-647.

Taheri, A. \& Zhalebaghi, M. H. (2017). A new model predictive control algorithm by reducing the computing time of cost function minimization for NPC inverter in three-phase power grids, ISA Transactions, 71(Pt 2), 391-402.

Xiao, W., Gao, Y. Wei, W. Zhang, J. \& Tan, X. (2021). Adaptive climbing motion of snake robot based on joint decoupling and extended Kalman filter, Journal of Physics: Conference Series, 1754(1), 012152 (6 pp).

Xu, K., Li, H. L. \& Liu, S. C. (2014). Speed Sensorless Control with ANN-MRAS Based on Modified ACO for Induction Motor Drives, Applied Mechanics and Materials, 705, 341-344. DOI: 10.4028/www. scientific.net/AMM.705.341

Yin, Z. Li, G., Sun, X., Jing, L. \& Zhong, Y. (2016). A speed estimation method for induction motors based on Strong Tracking Extended Kalman Filter. In 2016 IEEE 8th International Power Electronics and Motion Control Conference (IPEMC-ECCE Asia), 22-26 May (798 - 802).

Zaky, M. S. (2012). Stability Analysis of Speed and Stator Resistance Estimators for Sensorless Induction Motor Drives, IEEE Transactions on Industrial Electronics, 59(2), 858-870. DOI: 10.1109/ TIE.2011.2161658

Zhang, M. Cheng, M. \& Zhang, B. (2018) Sensorless Control of Linear Flux-Switching Permanent Magnet Motor Based on Improved MRAS. In 2018 IEEE 9th International Symposium on Sensorless Control for Electrical Drives (SLED), (pp. 84-89). DOI: 10.1109/ SLED.2018.8486093

Zhang, W., Ma, H. \& Gao, R. (2018). Rotor current speed sensorlesscontrol of doubly-fed induction generator based on proportional resonant regulator, Dianli Xitong Baohu yu Kongzhi/Power System Protection and Control, 46(10), 108-112. DOI: 10.7667/PSPC170748

https://www.sic.ici.ro 